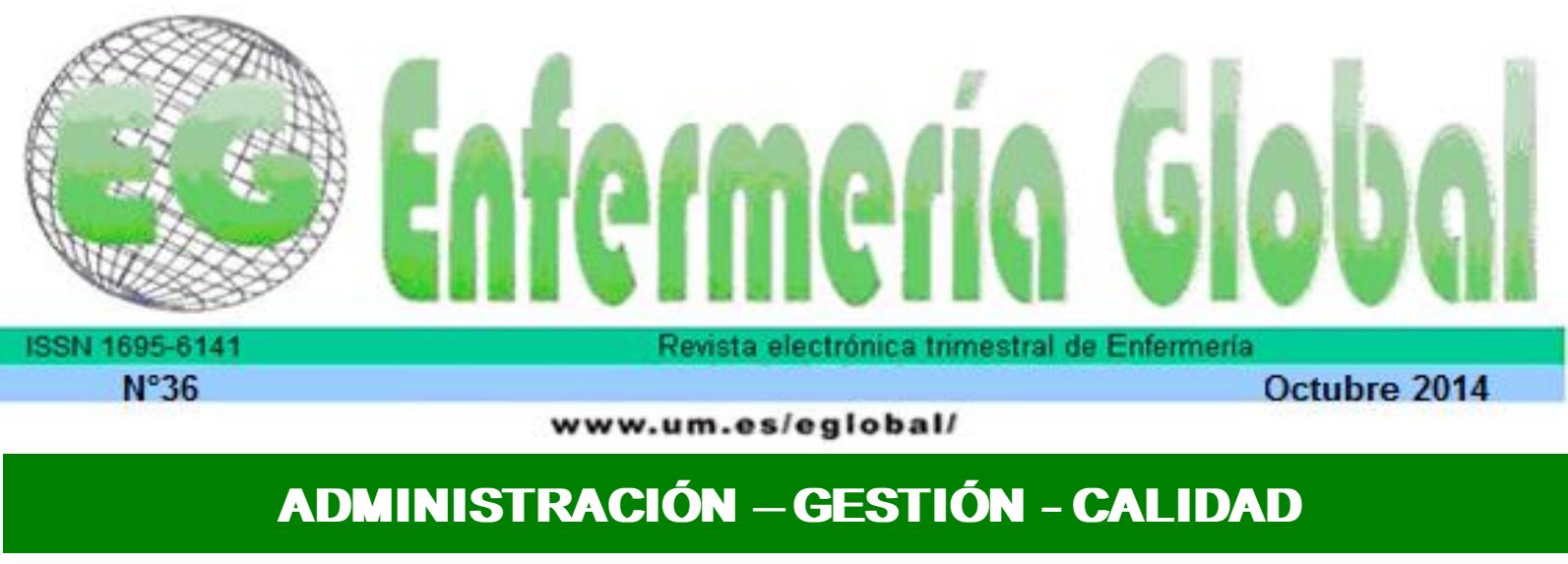

\title{
La investigación cualitativa en enfermería en Colombia (1990-2010)
}

Qualitative Research in nursing in Colombia (1990-2010)

\section{*Duque Páramo, María Claudia *Castellanos Soriano, Fabiola **Buitrago García, Carolina}

*Facultad de Enfermería. Pontificia Universidad Javeriana. E-mail: mcduque@javeriana.edu.co **Coordinadora de Gestión de Conocimiento. División de Investigaciones. Fundación Universitaria Ciencias de la Salud - FUCS. Colombia.

Palabras clave: Investigación Cualitativa; Investigación en Enfermería; Investigación Metodológica en Enfermería; Enfermería; Colombia.

Keywords: Qualitative Research; Nursing Methodology Research; Nursing Research; Nursing; Colombia.

\section{RESUMEN}

Presentamos un análisis sobre la Investigación Cualitativa (IC) en enfermería en Colombia, realizado con base en el diseño de una investigación cualitativa tipo estudio de caso, que incluyó como herramientas centrales entrevistas a informantes claves y de manera complementaria, revisión documental. Los datos se recolectaron de manera paralela entre junio y octubre de 2011. Se realizaron 19 entrevistas semiestructuradas a investigadores de universidades colombianas que han sido actores centrales en la IC en enfermería en Colombia y se revisaron 159 documentos publicados en revistas colombianas y de América Latina durante el período 1990 a 2010. El análisis interpretativo incluye como temas: los inicios de la IC en enfermería; las temáticas, abordajes y poblaciones; las contribuciones; las limitaciones y los retos para el futuro de la IC en enfermería en Colombia. A través de la práctica de la IC, las enfermeras colombianas han planteado críticas a los modelos biologicistas y positivistas hegemónicos de la investigación en salud; han realizado estudios con diversas personas y grupos poblacionales, han utilizado diversos enfoques y métodos, siendo la etnografía, la fenomenología, la teoría fundamentada y la IC los de mayor frecuencia, y han tenido limitaciones relacionadas con la calidad y el rigor, la falta de reconocimiento, la escasez de tiempo y dificultades para la financiación y la publicación. A pesar de las limitaciones, han contribuido a dar una mirada compleja a la humanización del cuidado de enfermería y trabajan en lograr un mayor impacto de las investigaciones en las políticas y acciones sociales.

\section{ABSTRACT}

In this article, we present an analysis of the Qualitative Research (QR) in nursing in Colombia, which was based on a qualitative case study design that included key informants interviews complemented by document review. Data were collected in a parallel manner between June and October 2011. We 
conducted 19 semi-structured interviews with researchers of Colombian universities that have been central actors in the QR nursing in Colombia and we reviewed 159 documents published by Colombian nursing researchers between 1990 and 2010 mainly in Colombian and Latin American journals. The interpretative analysis that we present include the following themes: the beginnings of QR in nursing in Colombia; the topics, approaches and populations; and finally, the contributions, limitations, and challenges for the future. Through their practice on QR Colombian nurses have raised criticisms of the hegemonic positivist and biologists models of health research studies; they have conducted research studies with various individuals and population groups; they have used different approaches and methods such as ethnography, phenomenology, grounded theory, among the ones with higher frequency; they have had limitations related to the quality and rigor, lack of recognition, lack of time, and difficulties in funding and publications. Despite these limitations, Colombian nurses conducting QR have helped to give a complex look and humanization of nursing care and currently work in achieving greater impact of their research on policies and social action.

\section{INTRODUCCIÓN}

En el campo de la salud y en la enfermería, es creciente el valor y el uso de la Investigación Cualitativa (IC) como una herramienta para conocer de manera comprensiva los aspectos subjetivos de la salud y la enfermedad a través del estudio de los significados, los saberes, las experiencias, vivencias y las perspectivas de las personas y colectivos con quienes trabajamos ${ }^{1-5}$. De manera semejante a lo ocurrido en otras disciplinas del área de la salud y acorde al énfasis hasta los años 80 en el estudio de las enfermedades, la investigación en enfermería en América Latina y en Colombia, durante la mayor parte del siglo pasado, utilizó métodos de corte objetivista centrados en la medición y en temas biomédicos y clínicos, es decir, se hizo en su mayoría desde paradigmas post-positivistas con un énfasis en las enfermedades y los individuos enfermos en contextos hospitalarios ${ }^{6-9}$.

En Colombia, a partir de los 1990s, el foco de la investigación se amplió al estudio comprensivo e interpretativo de los sujetos del cuidado, a "hacer realidad la voz del otro, paciente, cliente, comunidad" 6 , a través de los estudios cualitativos. Esta apertura ha estado relacionada con el interés de las enfermeras en el desarrollo de un campo disciplinar propio centrado en el cuidado de enfermería, el reconocimiento de la complejidad de la realidad y una crítica e insatisfacción con los modelos positivistas ${ }^{10}$. Así mismo, la capacidad de los estudios cualitativos de producir un conocimiento científico que propicia un espacio legítimo para las voces de los sujetos del cuidado, fortalece la práctica de la enfermería y contribuye al desarrollo disciplinar ${ }^{11}$.

El antecedente inicial del presente artículo es la invitación que hiciera Cheryl Beck, editora del Routledge International Handbook of Qualitative Nursing Research a investigadoras de Brasil, Chile, Colombia y México para escribir un capítulo sobre la Investigación Cualitativa (IC) en enfermería en América Latina. Derivada de esta invitación las autoras diseñamos una investigación cualitativa con el objetivo de describir las características y las tendencias de la IC en enfermería en Colombia entre 1990 y 2010; además de contribuir con el capítulo del libro sobre cuatro países de América Latina ${ }^{12}$, escribimos el presente artículo. Aunque ambos escritos comparten elementos conceptuales, metodológicos y de análisis, en este artículo discutimos en mayor detalle el panorama específico de la IC en enfermería en Colombia con énfasis en la perspectiva de los investigadores a quienes entrevistamos. En este sentido, el análisis que presentamos a continuación se enfoca en este aspecto particular, al mismo tiempo que revela lo que los participantes en la investigación plantearon sobre las contribuciones, las limitaciones y los desafíos relacionados con la formación, el rigor, la práctica y la toma de decisiones en salud. 


\section{MÉTODOLOGÍA}

Con el fin de cumplir con el objetivo, seguimos un diseño cualitativo con un enfoque de estudio de caso en el que combinamos entrevistas a informantes claves y revisión documental. Tal y como lo plantean algunos investigadores en el contexto de la IC y la IC en enfermería, el estudio de caso, como un abordaje o diseño de investigación cualitativa, puede ser utilizado de diferentes formas ya sea para el análisis de uno o de múltiples casos e incluye de manera característica el uso de fuentes de información y técnicas diversas tanto cualitativas como cuantitativas ${ }^{13,14}$

En este sentido, para esta investigación buscamos en primera instancia conocer y describir la perspectiva de los enfermeros que han sido actores en la producción de la IC en enfermería en Colombia a través de las entrevistas. De manera complementaria y como forma de triangulación de fuentes, utilizamos la revisión documental, vista como una técnica ${ }^{15}$, que, en este caso, al combinarla con las entrevistas, fortalece la calidad del análisis al reforzar y complementar lo planteado en las entrevistas

Las entrevistas y la revisión documental se realizaron de manera paralela entre junio y octubre de 2011. El primer acercamiento a las investigadoras-participantes, lo establecimos a través de las 37 decanas o directoras de facultades o de programas de enfermería afiliados a la Asociación Colombiana de Facultades de Enfermería (ACOFAEN), a quienes les enviamos cartas de invitación por medio del correo electrónico; éste también fue el medio de comunicación con los participantes a lo largo del estudio. A partir de sus respuestas, enviamos invitación a las investigadoras en cada institución.

En total respondieron 19 participantes que, antes de ser entrevistadas, estuvieron de acuerdo con el consentimiento informado. Éste fue aprobado por el Comité de Investigación y Ética de la Facultad de Enfermería de la Pontificia Universidad Javeriana y contempló todos los aspectos éticos considerados en la Resolución 008430 de 1993 y que incluyen los beneficios, riesgos, privacidad, anonimato y confidencialidad.

Luego de enviarles la guía de la entrevista, realizamos 19 entrevistas semiestructuradas con enfermeros-investigadores de 15 universidades del país. Las entrevistas se llevaron a cabo, en su mayoría, a través de Skype, en dos casos por teléfono y dos presencialmente en Bogotá. Los participantes tenían formación en maestría, doctorado o estudios de postgrado en curso; algunos ejercían cargos en las direcciones de investigación y en su mayoría contaban con amplia experiencia en IC o en ambas tradiciones metodológicas: cuantitativa y cualitativa. Las entrevistas fueron grabadas digitalmente y transcritas de manera textual con el fin de preparar los datos para los tres momentos del análisis cualitativo propuestos por Coffey y Atkinson: descriptivo, analítico e interpretativo ${ }^{16 .}$

Para la revisión documental se realizaron búsquedas en las bases de datos Index de Enfermería, Lilacs, Redalyc y Google Scholar. La búsqueda incluyó artículos durante el periodo comprendido entre 1990 y 2010, en los que era evidente el uso de la IC como diseño metodológico o como análisis conceptual. Las palabras clave utilizadas para la búsqueda fueron: enfermería, investigación cualitativa, nursing research, qualitative research y Colombia. Adicionalmente, se realizó una búsqueda detallada 
en las revistas de las facultades de enfermería del país en bibliotecas y a través de Internet, en revistas nacionales como Colombia Médica y la Revista de Salud Pública, y en la Revista de Salud Pública de México. Los documentos fueron registrados en una base de datos de Access que incluyó campos con la identificación del documento y las características de la investigación.

El análisis descriptivo de las entrevistas se realizó con matrices en Word; para la codificación inicial utilizamos las categorías deductivas y en un segundo momento categorías inductivas a partir de los datos. El momento analítico lo trabajamos con las matrices relacionando las categorías propuestas. En el momento interpretativo, nuestras voces se tejen con las de los participantes, la revisión documental y otros autores, para presentar nuestra interpretación sobre la IC en enfermería en Colombia entre 1990 y 2010. (Con fines éticos y de fortalecer la calidad de la investigación, compartimos con los participantes para su validación, una versión anterior del análisis. Este artículo incluye esta retroalimentación). Las voces de los participantes fueron sometidas a un Proceso de Transcreación, que consistió en editar errores gramaticales y palabras sin carga semántica, para hacer el texto más claro y comprensible $^{17}$.

\section{RESULTADOS}

Presentamos el análisis estructurado con los siguientes temas: inicios de la IC en enfermería; temáticas, abordajes y poblaciones; contribuciones; limitaciones; e interpretaciones finales y retos para el futuro de la IC en enfermería en Colombia.

\section{Los inicios de la investigación cualitativa en enfermería en Colombia}

De manera semejante al desarrollo de la enfermería en los Estados Unidos de América (EUA) y de otros países de América Latina, la investigación en enfermería en Colombia ha pasado por diversos momentos y métodos. Inicialmente y hasta los $60 \mathrm{~s}$ se enfocó en estudios sobre la enfermería y hasta la década de los 80s hubo un énfasis en estudios clínicos e investigaciones cuantitativas ${ }^{6,8,9}$. Paralelo a lo sucedido en América Latina $^{10}$, en Colombia a partir de los 90 s han ido en aumento el número de los estudios cualitativos sobre las experiencias de las personas (Tabla I).

Tabla I. Año de publicación de los documentos revisados sobre investigación cualitativa en enfermería en Colombia 1990 - 2010

\begin{tabular}{|c|c|c|}
\hline Año de Publicación & Frecuencia & Porcentaje \\
\hline $1991-1995$ & 5 & 3,1 \\
\hline $1996-2000$ & 18 & 11,3 \\
\hline $2001-2005$ & 28 & 17,6 \\
\hline $2006-2010$ & 108 & 67,9 \\
\hline Total & 159 & 100 \\
\hline
\end{tabular}

Fuente. Elaboración de las autoras

En el contexto de la enfermería colombiana, dos factores determinaron e hicieron posible el aprendizaje y la práctica de la IC: la formación de docentes universitarios en posgrados de Enfermería, Educación, Ciencias Sociales y Salud Pública en Estados Unidos y Brasil, y la inmigración de enfermeras extranjeras ${ }^{12}$. En el primer caso, al volver a Colombia, las docentes enriquecieron los programas de maestría y doctorado 
en instituciones como la Universidad Nacional de Colombia, la Universidad de Antioquia, la Universidad del Valle, la Pontificia Universidad Javeriana y la Universidad de la Sabana.

Posteriormente, otras docentes se vincularon como estudiantes a los programas de posgrado nacionales, adoptando la IC, al volver a sus instituciones. Esta forma de aprendizaje ha promovido el desarrollo de la IC en otras universidades de ciudades capitales que tienen influencia regional, como por ejemplo la Universidad del Cauca en Popayán, la Universidad Pedagógica y Tecnológica de Colombia en Tunja, la Universidad de Cartagena en Cartagena y la Universidad Francisco de Paula Santander en Cúcuta ${ }^{12}$. Experiencias regionales en investigación participativa comunitaria realizadas por docentes de la Universidad Pedagógica y Tecnológica de Colombia, también contribuyeron al surgimiento de la IC en enfermería ${ }^{18 .}$

La enfermera española Carmen de la Cuesta Benjumea, fue reconocida ampliamente entre los participantes como una de las pioneras en IC en Colombia. Además de las investigaciones que realizó en la Universidad de Antioquia y de artículos sobre temas metodológicos ${ }^{11,19,20}$, promovió el conocimiento mediante cursos, diplomados, traducción al español de libros especializados ${ }^{16,21-23}$ y la asesoría de trabajos de grado.

Aunque la educación formal fue determinante en el inicio de la IC, también contribuyeron otros factores como el cambio en los currículos de algunas universidades, el mayor acceso a publicaciones sobre la metodología cualitativa, el apoyo de otros investigadores experimentados, en muchas ocasiones provenientes de las ciencias sociales y humanas, y la participación de enfermeros con experiencia investigativa en estudios de corte cualitativo.

Cuando terminé la maestría me llamaron para que participara en una investigación; aprendí investigación cualitativa y me nutrí de su experiencia. (E 5).

Muchas enfermeras han trabajado con antropólogas y con sociólogos; en la facultad de enfermería... tenemos una cercanía muy grande con las ciencias humanas. (E 2).

Otro factor que impulsó la IC en Enfermería en Colombia se encuentra en las características personales de las investigadoras. La pasión y el interés por aprender han sido importantes, porque para hacer IC se requiere de una perspectiva diferente de pensamiento y luchar contra la mirada cuantitativa predominante.

Para mí la investigación es una pasión y un deseo; la hice muchos años [pagando] de mi bolsillo. (E 1).

Comencé a leer los trabajos... y de cómo de esas investigaciones se podían desprender intervenciones del cuidado de la enfermería, así ¡me apasioné por el tema! (E 7).

El auge de la IC en muchas facultades de enfermería ha ido de la mano de una crítica a la instrumentalización de la práctica de enfermería y de un interés en el estudio del cuidado como la categoría que define el quehacer profesional. Así mismo, se ha relacionado con algunas investigadoras que, con una postura crítica frente al poder y 
la hegemonía de la práctica de enfermería desde la biomedicina, se han interesado en realizar investigaciones participativas sobre las perspectivas de los actores sociales con menos poder en la sociedad colombiana como son: mujeres, ancianos, niños, discapacitados o personas en situación de desplazamiento ${ }^{12}$.

\section{Temáticas, abordajes y poblaciones}

De acuerdo a lo expresado por los participantes y a la revisión documental, las principales temáticas de las investigaciones son acerca de los significados y las experiencias en situaciones de salud y enfermedad. Éstas incluyen estudios con personas con cáncer, o con enfermedades crónicas en diversos contextos: unidades de cuidado intensivo, áreas de hospitalización, comunidades rurales, urbanas y marginales e instituciones educativas ${ }^{20,24-27}$. Le siguen investigaciones sobre los aspectos culturales del cuidado y el estudio de la diversidad de saberes, actitudes y prácticas de la salud y la enfermedad ${ }^{18,28-30}$. Un tercer conjunto de temas se refiere a las experiencias y significados del cuidado en gestantes y puérperas ${ }^{31,32}$ y en los cuidadores $^{19,33,34}$. Otros estudios se enfocaron en aspectos particulares de los roles y el quehacer en enfermería ${ }^{35,36}$.

La IC comprende una gran diversidad de enfoques y métodos originados en disciplinas de las ciencias sociales y humanas, cuyo propósito central es comprender e interpretar la realidad desde la perspectiva de los actores sociales. Aunque en general se incluye a la IC dentro del paradigma constructivista, no hay un paradigma o teoría única que la caracterice, sino que tiene su origen en perspectivas teóricas tan diversas como el constructivismo, la hermenéutica, los estudios culturales, el feminismo, el marxismo y el interaccionismo simbólico. Así mismo quienes la practican utilizan variedad de enfoques metodológicos y estrategias de análisis, entre los que se destacan la etnografía, la fenomenología y la teoría fundamentada y herramientas como entrevistas, observación participante, fotografías y dibujos y revisión documental entre muchos otros ${ }^{37-39}$

De manera semejante a lo que han destacado algunos estudiosos de la IC en Salud en lberoamérica ${ }^{10}$, en el diseño de la investigación los enfermeros colombianos utilizan diversos denominaciones, métodos y formas de abordajes. La mayor proporción de los artículos revisados correspondió a lo que algunos autores llamaron estudio cualitativo y otros denominaron investigación comprensiva, descriptiva o participativa $^{18,40,41}$. Estas investigaciones las categorizamos de una manera genérica como estudios cualitativos. Les siguen los diseños etnográficos -nombrados como etnografía solamente, o con adjetivos como etnografía descriptiva, focalizada, particularista o reflexiva, y microetnografía ${ }^{26,42,43}$; los diseños fenomenológicos, también nombrados fenomenologías ${ }^{44-46}$; de teoría fundamentada o teoría fundada ${ }^{47}$, ${ }^{48}$ y en menor proporción, la etno-enfermería ${ }^{28,32}$. 
Tabla II. Tipo de diseños con base en la denominación que dan los autores en los artículos publicados sobre investigación cualitativa en enfermería en

Colombia 1990-2010

\begin{tabular}{|l|c|c|}
\hline \multicolumn{1}{|c|}{ Tipo de Diseños } & Frecuencia & Porcentaje \\
\hline Etnográficos & 37 & 23,3 \\
\hline Cualitativos & 36 & 22,7 \\
\hline Fenomenológicos & 30 & 18,9 \\
\hline Teoría fundamentada & 17 & 10,7 \\
\hline Participativos & 8 & 5 \\
\hline Otras denominaciones & 31 & 19,5 \\
\hline Total & 159 & 100 \\
\hline
\end{tabular}

Fuente. Elaboración de las autoras

La población sujeto de las investigaciones, en mayor proporción fueron los colectivos: cuidadores, estudiantes de enfermería, indígenas, comunidades barriales y familias; le siguieron las investigaciones con mujeres y luego con adultos en diversas situaciones de salud: hospitalizados, con VIH, con discapacidades y con enfermedades agudas o crónicas como el cáncer. La menor proporción se realizó con adolescentes, ancianos y niños.

Los temas, los abordajes y las poblaciones con quienes trabajaron los investigadores, estuvieron determinados por tres factores: las líneas de trabajo asumidas desde la formación de posgrado de las investigadoras, quienes intentan mantener el área temática, la metodología y la población de estudio con las que trabajaron durante sus estudios; el segundo, los intereses de las investigadoras en explorar otras dimensiones del cuidado de enfermería, y el tercero las principales situaciones y problemas de salud en el país, que también se relacionan con los intereses y políticas de financiación de organismos nacionales e internacionales.

\section{Contribuciones de la investigación cualitativa en enfermería en Colombia}

La práctica de la IC en Colombia ha contribuido al fortalecimiento de la profesión, a la visibilidad de la enfermería en la comunidad académica y ha aportado beneficios a los sujetos del cuidado. Desde el interior de la profesión, la labor investigativa con diseños cualitativos ha permitido a los enfermeros colombianos transitar de una mirada predominantemente biologicista y patocéntrica, a una mirada centrada en el sujeto de cuidados desde una perspectiva social. Relacionado con que, como lo plantea Morse "nuestros métodos son humanísticos, gentiles y amables, en interacción empática con nuestros participantes" 5 , la IC es reconocida por los participantes, como una herramienta valiosa para explorar, comprender y reconocer las realidades propias del cuidado de enfermería, de la condición humana y del cuidado de la salud. En la medida que la IC permite ir más allá de los aspectos biológicos, haciendo visibles la complejidad de las realidades de los sujetos, ha aportado a la profesión y a la humanización de la práctica y el cuidado de enfermería.

El principal aporte es humanizar el cuidado y reconocer que el otro es muy importante. (E 16).

Puesto que la IC promueve relaciones basadas en la participación y ayuda a la catarsis y expresión de sentimientos, su práctica ha contribuido a cuestionar los estilos jerárquicos y autoritarios propios de la biomedicina y a promover el empoderamiento de diversos actores sociales. Además de los beneficios emocionales 
para los participantes, en algunos casos, como resultado de las investigaciones se han diseñado programas y acciones ajustados a las realidades y perspectivas de las personas y los colectivos.

La IC también ha propiciado reestructurar la enseñanza de la enfermería colombiana y fortalecer la investigación. En algunas instituciones se han introducido cambios en los currículos sobre el aprendizaje y la práctica de la IC en el pregrado y el posgrado y se incentiva la IC en semilleros de investigación y en la formulación de trabajos de grado.

La IC también ha sido fuente de empoderamiento para las enfermeras, quienes tienen reconocimiento entre la comunidad académica nacional, por su competencia como investigadoras y pioneras en el uso de la IC en Colombia y en las ciencias de la salud. Otra contribución ha sido sembrar el interés en algunas investigadoras en generar teorías propias que contribuyan en la solución de problemas prácticos y al desarrollo de la disciplina.

\section{Limitaciones en el desarrollo de la investigación cualitativa en enfermería en Colombia}

Además de los logros, los participantes plantearon limitaciones en la formación de estudiantes e investigadores; problemas relacionados con la calidad, el rigor y la falta de reconocimiento de la IC dentro y fuera de la enfermería; restricciones de tiempo y dificultades para la financiación y la divulgación. También resaltaron el escaso impacto de la IC en la práctica de la enfermería y en la toma de decisiones y el diseño de políticas públicas en salud.

Dentro de los factores que dificultan el aprendizaje y la apropiación de la IC en los estudiantes, se encuentran: limitadas habilidades de lectura y escritura, desinterés de algunos estudiantes y profesores y un estilo pedagógico memorístico que no propicia el pensamiento crítico y sistemático. La falencia de algunos de estos elementos centrales para el aprendizaje de la IC, ya documentada desde inicios de los $90 \mathrm{~s},{ }^{49}$ continúa presente.

La IC exige un ejercicio mayor de lectura y escritura; esa es una de las grandes dificultades. El estudiante...lee sin comprender $y$ no hay capacidad interpretativa. (E 1).

Otra limitante se encuentra en la hegemonía del modelo positivista en la enseñanza de los programas de enfermería, que promueve una relación monológica, jerárquica y autoritaria basada en la obediencia, la cual es contraria al diálogo, la participación, el poder compartido con el sujeto de cuidado y las relaciones horizontales, que requiere la IC. A pesar de las críticas, algunas personas se resisten a abrirse hacia otras perspectivas.

Mucha gente se queda con el enfoque positivista y hacer el cambio es como un salto al vacío... a lo desconocido... y esos reaprendizajes no todos están dispuestos a hacerlos (E 5).

Otras limitaciones expresadas por los participantes tienen que ver con el poco interés y la escasa formación de algunos profesores y estudiantes en otras disciplinas, en conceptos y herramientas cualitativas y en el inglés. La falta de interés la relacionan 
con que la IC demanda una gran dedicación en tiempo y compromiso que además del trabajo de campo incluye el análisis, la interpretación, la validación y devolución de la información a los participantes.

Aunque la actualización solucionaría la falta de formación de los docentes, las limitaciones financieras obstruyen el camino a muchos profesores que no se actualizan y como tienen poca experiencia en IC, sienten temor de asumirla, lo cual limita su uso. Adicionalmente, no todos tienen acceso a la capacitación en herramientas para la sistematización y el análisis, lo que influye en el rezago de algunos investigadores y en problemas en la calidad de la IC.

Relacionadas con las debilidades en la formación, algunos participantes resaltan la escasa fundamentación epistemológica y metodológica de algunos investigadores y la baja calidad y poco rigor de algunas publicaciones que tienen problemas como: escasa documentación de los aspectos metodológicos, poco diálogo con otros autores, poca rigurosidad en el análisis de los datos, desconocimiento de estrategias de análisis específicas de la IC o limitada interpretación.

Hemos encontrado investigaciones cualitativas en enfermería que no soportan una mirada crítica a la metodología, en los artículos no se dice cómo se hizo la investigación... se limitan a decir que se hicieron tantas entrevistas... y les falta fortaleza metodológica. (E 3).

Otras limitaciones tienen que ver con el poco reconocimiento y la falta de apoyo entre los colegas, problemas en la evaluación de pares y desconocimiento del valor de la IC y de criterios aceptados por las comunidades académicas para juzgar su calidad. Éstas se relacionan con que todavía una parte de la comunidad en enfermería y en el área de la salud, desconoce las características, los usos y cómo juzgar la calidad de la IC. Esto, unido a la hegemonía y el mayor estatus de los enfoques positivistas, condiciona ya sea, a no reconocer, o, a reflejar una idea negativa de la IC. Como lo plantea De la Cuesta, hay quienes, desde perspectivas cuantitativas, consideran a la IC, una investigación suave basada en opiniones ${ }^{11}$ que no cumple con los criterios para ser considerada evidencia de calidad. Estos juicios impactan en el limitado número de proyectos con diseños cualitativos en enfermería que son financiados en convocatorias nacionales y condicionan la exclusión de la IC de fuentes de financiación que limitan el concepto de evidencia a la cuantitativa.

En las convocatorias, la cualitativa sigue llevando las de perder porque no nos ven como investigación... iprefieren las investigaciones duras! (E 2).

El problema de evaluadores sin formación ni sensibilidad hacia la IC se encuentra presente también en otras partes del mundo 1; en Colombia es posible encontrarlo en la evaluación que realizan los pares a proyectos de investigación y artículos, los cuales con alguna frecuencia son evaluados desde la mirada cuantitativa por personas que no conocen ni tienen experiencia en IC. Las limitaciones en cuanto al apoyo se relacionan, además de las ya mencionadas, con que muchas investigadoras trabajan solas, es decir, no cuentan con equipos de trabajo o redes en las cuales apoyarse.

El tiempo para dedicarse a la IC, preparar y gestionar las publicaciones es otra limitante importante para el avance y el reconocimiento. Aunque en las universidades en donde se desempeñan la mayoría de los participantes, cuentan con tiempos para 
la docencia y la investigación, éstos son insuficientes para realizar otras actividades como ampliar su formación en teorías y métodos, publicar y visibilizar los resultados para las comunidades académicas y el público en general y avanzar hacia la construcción de teorías de rango medio en enfermería.

Las limitaciones en la financiación de la IC están relacionadas con algunos aspectos ya planteados: el desconocimiento de la IC de parte de agencias financiadoras y pares académicos, la baja calidad de algunos proyectos, la falta de tiempo de los investigadores para mejorar su formación y para centrarse en la escritura, y la hegemonía del paradigma positivista.

La divulgación de la mayoría de los estudios en IC en enfermería en Colombia se hace a través de las revistas de las facultades de Enfermería. Algunas de ellas tienen clasificaciones de alta calidad; sin embargo, son pocas y en ocasiones los tiempos de espera para la publicación pueden llegar a ser largos. Las enfermeras colombianas también publican en revistas de otras profesiones o de otros países, en general en revistas en español o portugués y algunas pocas han publicado en revistas de idioma inglés.

\section{DISCUSIÓN}

Los enfermeros colombianos que desde la década de los 90 adoptaron la IC como forma de producción de conocimiento válido, han planteado críticas a los modelos biologicistas y positivistas, hegemónicos en la investigación en salud ${ }^{7,50}$ y a formas instrumentalistas y subordinadas de la práctica de enfermería. En la discusión planteamos la interpretación uniendo nuestras voces con las de los participantes y las de algunos de los otros pocos investigadores sobre IC en enfermería en Latinoamérica y España.

Desde sus inicios en los 90s, la IC en enfermería en Colombia ha buscado profundizar en el conocimiento sobre los aspectos culturales y los sujetos del cuidado, así como de sus experiencias y significados en diversas situaciones de salud y enfermedad en contextos hospitalarios y comunitarios $y$ en zonas urbanas y rurales. Los investigadores han utilizado enfoques, métodos y herramientas como la etnografía, la fenomenología, la teoría fundamentada, los diseños participativos, y la investigación cualitativa en salud.

Como tendencia general, los participantes explicaron con razones personales o gremiales las limitaciones que identificaron en la formación, el rigor, la disponibilidad de tiempo y de recursos financieros, la divulgación y el impacto y reconocimiento de la IC. Sin embargo, consideramos necesario tener en cuenta que estas dificultades no son exclusivas de la IC en Colombia, sino que también han sido reconocidas en la investigación en enfermería en otras partes del mundo 1, 9, 10, 51-53. Adicionalmente y, de manera semejante a lo que sucede en otros países de América Latina ${ }^{7}$, en Colombia los desarrollos desiguales de la IC en enfermería se relacionan con marcadas inequidades sociales y económicas estructurales del país. En el campo de la enfermería, éstas se expresan, entre otras, en inequidades en el acceso a la educación superior en enfermería ${ }^{54}$. En este sentido, consideramos que más allá de las características personales o gremiales, las limitaciones se relacionan con inequidades en el acceso a saberes y a recursos para desarrollar investigación de calidad. 
En este contexto, nos unimos ${ }^{12}$ a los participantes para recomendar: fortalecer la capacidad de investigadores y estudiantes en los diseños, el análisis y el rigor de la IC; promover alianzas regionales, nacionales e internacionales con el fin de realizar estudios multicéntricos, intercambios de profesores y estudiantes y aprendizaje e innovación de experiencias; y avanzar hacia la construcción de modelos y teorías que desde nuestras realidades contribuyan al desarrollo disciplinar de la enfermería mundial. La IC provee a los enfermeros de posibilidades reales para generar teorías y conocimientos para comprender los fenómenos y aportar diferentes formas de pensar el cuidado de la salud y la vida. En este sentido, la evidencia cualitativa hace una contribución disciplinar que permite repensar el mundo que nos rodea y suscitar preguntas cuyas respuestas orientan la práctica ${ }^{50}$.

\section{CONCLUSIONES Y RETOS}

Los enfermeros que en Colombia han utilizado a la IC como forma de producción de conocimiento, han trabajado con diversas poblaciones, promoviendo una mirada compleja de la realidad y la humanización del cuidado de enfermería. Para ello han utilizado una amplia variedad de enfoques y métodos, que incluyen diseños de amplia trayectoria como la etnografía, la teoría fundamentada, la investigación cualitativa en salud y la fenomenología.

Así mismo a través de la práctica de la IC han logrado un impacto positivo en los programas de pregrado y posgrado en enfermería en Colombia, han contribuido al reconocimiento, la autonomía y el fortalecimiento disciplinar y al mismo tiempo han encontrado obstáculos, limitaciones y retos.

En este contexto, un reto de la mayor importancia es hacer que nuestras investigaciones tengan aplicabilidad y mayor impacto en las condiciones de vida de las personas y las comunidades con quienes trabajamos. En este sentido, consideramos prioritario: fortalecer y ampliar el uso de la evidencia cualitativa en el diseño de políticas, acciones y $\operatorname{programas}^{50}$; la retroalimentación y la entrega de informes a los participantes en las investigaciones; la apertura a los diseños mixtos con el fin de aportar evidencias cuantitativas y cualitativas para el diseño de programas y políticas de salud; y la implementación de herramientas que propicien mayor participación y construcción conjunta con los participantes, como por ejemplo metodologías basadas en el juego y el arte ${ }^{12}$.

\section{Agradecimientos}

La investigación base de este artículo fue financiada por la Pontificia Universidad Javeriana. Así mismo, agradecemos de manera especial a las investigadoras e instituciones que apoyaron y brindaron sus conocimientos y experiencias para realizar esta investigación.

\section{REFERENCIAS}

1. Beck CT. Introduction to qualitative nursing research. En: Beck CT, organizador. Routledge International Handbook of Qualitative Nursing Research. New York: Routledge, 2013. p. 1-10.

2. The PLoS Medicine Editors. Qualitative Research: Understanding Patients' Needs and Experiences. PLoS Med. 2007 Aug; 4(8):e258. Disponible en: http://dx.doi.org/10.1371\%2Fjournal.pmed.0040258. 
3. Morse JM. What is the domain of qualitative health research? Qual Health Res. $2007 \mathrm{Jul} ; 17(6): 715-7$.

4. Monteiro CFdS, Moreira MRC, Oliveira EAR, Moura MES, Costa JV. Pesquisaação: contribuição para prática investigativa do enfermeiro. Revista Gaúcha de Enfermagem. 2010 Mar; 31(1):167-74.

5. Morse JM. The development of qualitative nursing research. En: Beck CT, organizador. Routledge International Handbook of Qualitative Nursing Research. New York: Routledge, 2013. p. 13-31.

6. Duque-Páramo MC. Una mirada al desarrollo de la investigación en enfermería. Investigación en Enfermería Imágen y Desarrollo. 1999 Enero-Junio; 1(1):25-32. Disponible en: http://revistas.javeriana.edu.co/index.php/imagenydesarrollo/article/view/1168. 7. Do Prado ML, De Souza MdL, Carraro TE, Román de Cisneros G, Arzuaga MA. Producción del conocimiento en enfermería en América Latina: Un meta-análisis. En: Do Prado ML, De Souza MdL, Carraro TE, organizadores. Investigación cualitativa en enfermería: Contexto y bases conceptuales. Washington: Organización Panamericana de la Salud OPS, 2008. p. 1-15.

8. Castrillón Agudelo MC. Trends and priorities in nursing research. Rev Latinoam Enfermagem. 2004 Julho-Agosto; 12(4):583-8. Disponible en: http://www.scielo.br/scielo.php?pid=S0104-11692004000400002\&script=sci arttext.

9. Manfredi M. El desarrollo de enfermería en América Latina: una mirada estratégica. Rev Latino-Am Enfermagem. 1993 Jan; 1(1):23-35. Disponible en: http://www.scielo.br/scielo.php?pid=S0104-11691993000100004\&script=sci arttext.

10. Gastaldo D, Mercado-Martinez FJ, Ramasco-Gutierrez M, Lizardi-Gomez A, Gil-Nebot MA. Qualitative health research in Ibero-America: the current state of the science. J Transcult Nurs. 2002 April; 13(2):90-108. Disponible en: http://www.ncbi.nlm.nih.gov/entrez/query.fcgi?cmd=Retrieve\&db=pubmed\&dopt=Abstract\&list uids $=11951720$.

11. Cuesta Benjumea Cdl. La investigación cualitativa y el desarrollo del conocimiento en enfermería. Texto y Contexto Enferm. 2010 Out- Dez; 19 (4):762-6. Disponible en: $\quad$ http://www.scielo.br/scielo. php?pid=S0104$07072010000400020 \&$ script $=$ sci arttext.

12. Duque-Páramo MC, Padilha MI, Sanhueza Alvarado O, Alonso Castillo MM, Castellanos Soriano F, López García KS, et al. Qualitative Nursing Research in Latin America. The Cases of Brazil, Chile, Colombia and Mexico. En: Beck CT, organizador. Routledge International Handbook of Qualitative Nursing Research. New York: Routledge, 2013. p. 478-99.

13. Creswell JW. Five qualitative approaches to inquiry. En: Creswell JW, organizador. Qualitative inquiry and research design: choosing among five traditions. Thousand Oaks, CA: Sage, 2007. p. 53-84.

14. Holloway I, Wheeler S. Qualitative Research in Nursing and Healthcare. Oxford: Wiley-Blackwell, 2010.

15. Galeano Marín ME. Investigación documental: una estrategia no reactiva de investigación social. En: Estrategias de investigación social cualitativa El giro en la mirada. Medellín: La Carreta Editores, 2004. p. 113-44.

16. Coffey A, Atkinson P. Encontrar el sentido a los datos cualitativos. Medellín: Universidad de Antioquia, 2003.

17. Sebe Bom Meihy JC. Manual de historia Oral. 5 ed. Sao Paulo, Brasil: Ediciones Loyola, 2005.

18. Romero B. MN. El saber y las prácticas médicas tradicionales. Avances en Enfermería. $1995 \quad$ 13(2):71-6. Disponible http://www.revistas.unal.edu.co/index.php/avenferm/article/view/16503. 
19. Cuesta Benjumea Cdl. La artesanía del cuidado: cuidar en la casa a un familiar con demencia avanzada. Enfermería Clínica. 2005 Diciembre; 15(6):335-42. Disponible en: http://rua.ua.es/dspace/handle/10045/17223.

20. Cuesta Benjumea Cdl. Las mujeres y el manejo de un síntoma: de la valoración a la selección. Salud Pública de México. 1999 Marzo-Abril; 41(2):124-9. Disponible en: http://rua.ua.es/dspace/handle/10045/17221.

21. Strauss A, Corbin J. Bases de la investigación cualitativa. Técnicas y procedimientos para desarrollar la teoría fundamentada. Medellín: Universidad de Antioquia, 2002.

22. Morse JM. Asuntos críticos en los métodos de investigación cualitativa. Medellín: Universidad de Antioquia, 2003.

23. Wolcott HF. Mejorar la escritura de la investigación cualitativa. Medellín: Universidad de Antioquia, 2003.

24. Moreno Fergusson ME, Rodríguez MC, Gutiérrez Duque M, Ramírez LY, Barrera Pardo O. ¿Qué significa la discapacidad? Aquichan. 2006 Octubre; 6(1):7891. Disponible en: http://aquichan.unisabana.edu.co/index.php/aquichan/article/view/82/169. 25. Beltran-Salazar OA. Hay que poner de su parte para afrontar una enfermedad grave. Aquichan. 2010 Diciembre; 10(3):214-27. Disponible en: http://aquichan.unisabana.edu.co/index.php/aquichan/article/view/1740.

26. Duque-Páramo MC. No me gusta, pero está bien si me porto mal. Voces sobre el castigo de niñas y niños de un barrio de Bogotá. Investigación en Enfermería Imagen y Desarrollo. 2008 Enero-Junio; 10(1):113-34. Disponible en: https://www.academia.edu/205920/No me gusta pero esta bien si me porto mal. Voces sobre el castigo de ninas y ninos de un barrio de Bogota.

27. Álvarez C. LS. Las decisiones de los habitantes de Medellín en materia de servicios de salud. Invest Educ Enferm. 2008 Marzo; 26(1):98-105. Disponible en: http://www.redalyc.org/pdf/1052/105215231009.pdf.

28. Argote O. LÁ, Mejía R. ME, Vásquez T. ML, Villaquirán de González ME. Climaterio y menopausia en mujeres afrodescendientes una aproximacion al cuidado desde su cultura. Aquichan. 2008 Abril; 8(1):33-49. Disponible en: http://www.redalyc.org/articulo.oa?id=74108105.

29. Melguizo Herrera E, Lucía AM. Creencias y practicas de cuidado de la salud de ancianos. Avances en Enfermería. 2010 Octubre; 28:61-72. Disponible en: http://www.revistas.unal.edu.co/index.php/avenferm/article/view/21450.

30. Eslava Albarracín DG. Los agentes tradicionales de salud: otra alternativa de salud para las comunidades campesinas el caso de Cundinamarca y Santander. Investigación y Educación en Enfermería. 1998 Septiembre; 16(2):57-70. Disponible en: http://aprendeenlinea.udea.edu.co/revistas/index.php/iee/article/viewFile/16898/14630.

31. Soto VI, Durán de Villalobos MM. Experiencias y vivencias del embarazo en adolescentes explicadas desde lo social. Avances en Enfermería. 2008 JulioDiciembre; 26(2):98-106. Disponible en: http://revistas.unal.edu.co/index.php/avenferm/article/view/12944.

32. Bernal-Roldán MC, Lucy MdR, Ruiz de Cárdenas $\mathrm{CH}$. Significado del cuidado de sí y de su hijo por nacer en gestantes desplazadas. Aquichan. 2008 Abril; 8(1):97115. Disponible

en: http://aquichan.unisabana.edu.co/index.php/aquichan/article/view/127/255.

33. Buitrago-Echeverri MT, Ortiz-Rodríguez SP, Eslava-Albarracín DG. Necesidades generales de los cuidadores de las personas en situación de discapacidad. Investigación en Enfermería Imagen y Desarrollo. 2010 Enero-Junio; 12(1):59-77. Disponible en: http://www.redalyc.org/articulo.oa?id=145216903005.

34. Rodríguez Holguín YA MdRL. Las madres se adaptan a la hospitalización de sus hijos mediante la exploración y apoyo. Actualizaciones en Enfermería. 2009 
Marzo;

http://www.encolombia.com/medicina/enfermeria/Enfermvol120109/madresseadaptanalahospi talizacion.htm.

35. Vanegas C. BC, Vargas R. E, Abella M. LF, Alayon G. MY, Bonza S. LP, Chaparro D. EY, et al. Roles que desempeñan profesionales de enfermería en instituciones geriátricas de Bogotá. Revista Colombiana de Enfermería. 2010 Agosto; 5:81-92. Disponible en: http://bases.bireme.br/cgibin/wxislind.exe/iah/online/?IsisScript=iah/iah.xis\&src=google\&base=LILACS\&lang=p\&nextActi on=Ink\&exprSearch=616052\&indexSearch=ID.

36. Ospina Rave BE, Toro Ocampo JA, Aristizábal Botero CA. Rol del profesor en el proceso de enseñanza aprendizaje de la investigación en estudiantes de Enfermería de la Universidad de Antioquia, Colombia. Investigación y Educación en Enfermería. 2008 Marzo; 26(1):106-14. Disponible en: http://www.redalyc.org/articulo.oa?id=105215231010.

37. Creswell JW. Qualitative inquiry and research design: choosing among five traditions. Thousand Oaks, CA: Sage, 2007.

38. De Souza Minayo MC. El desafío del conocimiento: investigación cualitativa en salud. 8ํㅡ. ed. Buenos Aires: Lugar Editorial, 2004.

39. Galeano Marín ME. Estrategias de investigación social cualitativa. El giro en la mirada. Medellín: La Carreta Editores, 2004.

40. Lafaurie MM, Barón P. LV, León S. DC, Martínez M. PM, Molina Q. DC, Rodríguez V. DY, et al. Madres cuidadoras de niños(as) con cáncer: vivencias, percepciones y necesidades. Revista Colombiana de Enfermería. 2010 Agosto; 5:4152.

Disponible

en: http://www.uelbosque.edu.co/sites/default/files/publicaciones/revistas/revista colombiana enf ermeria/volumen5/madres cuidadoras ninos cancer.pdf.

41. Eslava Albarracín DG, Puntel de Almeida MC. Salud y enfermedad expresión gráfica de ideas en estudiantes de pregrado en enfermería. Invest Educ Enferm. 2005 Septiembre; 23(2):70-81.

Disponible

en: http://www.redalyc.org/articulo.oa?id=105215403006.

42. Castellanos Soriano F, López Díaz AL. Mirando pasar la vida desde la ventana: significados de la vejez y la discpacidad de un grupo de ancianos en un contexto de pobreza. Investigación en Enfermería Imagen y Desarrollo. 2010 Julio-Diciembre; 12(2):37-53. Disponible en: http://www.redalyc.org/articulo.oa?id=145217280004.

43. Salazar-Maya ÁM, Guarín-Berrío GE, Arroyave-Cadavid ML, Ochoa-Acosta JE, Galeano-Ochoa M. La higiene de las manos en una unidad de cuidado intensivo. Aquichan. $2008 \quad$ Abril; 8(1):8-18. Disponible en: http://aquichan.unisabana.edu.co/index.php/aquichan/article/view/120.

44. Morales LC, Castillo E. Vivencias de los(as) adolescentes en diálisis: una vida con múltiples pérdidas pero con esperanza. Colomb Med. 2007 Octubre-Diciembre; 38(4(Supl 2)):44-53. Disponible en: http://colombiamedica.univalle.edu.co/index.php/comedica/article/view/536/956.

45. Canaval GE, González MC, Tovar MC, Valencia C. La experiencia de las mujeres gestantes: "lo invisible". Investigación y Educación en Enfermería. 2003 Septiembre; $21(2): 32-46$. Disponible en: http://aprendeenlinea.udea.edu.co/revistas/index.php/iee/article/viewFile/3010/2752.

46. Zambrano Plata GE, Ferrera Cardona JC, Lindarte Clavijo AA, Niño Bayona CJ, Ramírez Rodríguez NR, Rojas Bautista LY. Entre la incertidumbre y la esperanza: Percepciones y motivaciones de los pacientes hospitalizados en la unidad de cuidados intensivos (UCI). Revista Ciencia y Cuidado. 2010 Enero-Diciembre; 7(1):5260. Disponible en: http://dialnet.unirioja.es/servlet/articulo?codigo=3717275. 
47. Cacante C. JV, Arias Valencia MM. Tocar los corazones en busca de apoyo: el caso de las familias de los niños con cáncer. Invest Educ Enferm. 2009 Septiembre; 27(2):170-80.

48. Alvarado García AM. Experiencia de cuidar a un paciente con enfermedad crónica después de recibir una capacitación. Salud Uninorte. 2010 Julio-Diciembre; 26(2):232-49.

Disponible

en:

http://rcientificas.uninorte.edu.co/index.php/salud/article/viewFile/221/834.

49. Romero Ballen MN. El Modelo Pedagógico en Enfermeria: una proyección del papel social de la mujer. En: Memorias del II Congreso Nacional De Patógenos; Tunja, Boyacá: Universidad Pedagógica y Tecnológica de Colombia; 1991. p. 15-22.

50. Cuesta Benjumea Cdl. La contribución de la evidencia cualitativa al campo del cuidado y la salud comunitaria. Index Enferm. 2005 Otoño; 14(50):47-52. Disponible en: http://rua.ua.es/dspace/handle/10045/18358.

51. Cabral IE, Tyrrel MAR. Pesquisa em enfermagem nas Américas. Rev Bras Enferm. $2010 \quad$ Jan-Fev; 63(1):104-10. Disponible en: http://www.scielo.br/scielo.php?pid=S0034-71672010000100017\&script=sci arttext.

52. Lange I, Leonhardy $\mathrm{K}$. The need for evidence in nursing practice in Latin America. Applied Nursing Research. 2006 November; 19(4):220-1. Disponible en: http://www.sciencedirect.com/science? ob=GatewayURL\& origin=ScienceSearch\& method= citationSearch\& piikey=S0897189706000954\& version=1\& returnURL=http\%3A\%2F\%2Fww w.scirus.com\%2Fsrsapp\%2F\&md5=6982f8fc6fb2870c619ef029101baaac.

53. Mendoza-Parra S, Paravic-Klijn T, Muñoz Muñoz AM, Barriga OA, JiménezContreras E. Visibility of Latin American Nursing Research (1959-2005). Journal of Nursing Scholarship. 2009 March; 41(1):54-63.

54. Duque-Páramo MC, López Maldonado MC. Nursing in Colombia. En: Breda KL, organizador. Nursing and Globalization in the Americas A critical perspective. Hartford: Baywood Publishers, 2009. p. 21-54. 